\title{
Escala de atitudes frente ao HIV/AIDS: análise de fatores
}

\author{
HIV/AIDS attitudes scale: factorial analysis
}

Nelson Silva Filho', Pedro Henrique Godinho', César Henrique dos Reis', Nádia Maria Silva Pacheco

\section{RESUMO}

Objetivo: Este trabalho apresenta resultados acerca das propriedades psicométricas da "Escala de atitudes frente ao HIV/AIDS". Os dados, provenientes de uma amostra de 549 alunos entre universitários, ensinos médio e ensino fundamental. Métodos: Os dados foram tratados pelo método dos componentes principais da análise fatorial. A análise final, postulado um eigenvalue mínimo de 2, resultou cinco fatores. Foram eliminados itens que apresentaram carga fatorial menor que 0,30. Neste estudo, o menor alfa observado foi de 0,79. Portanto, é provável que todos os 47 itens do instrumento final elaborado meçam o mesmo construto: atitude frente ao HIV/AIDS. Resultados: Escores inferiores a 96 foram considerados "fraco grau de conhecimento sobre HIV/AIDS"; entre 96 e 192 "moderado grau de conhecimento" e acima de 192 "alto grau de conhecimento sobre HIV/AIDS". Foram estabelecidos os fatores: 1, 2 e 3, sendo "fator geral de percepção da informação técnico-científica"; "fator de percepção da informação técnico-científica versus sexualidade e preconceito"; "fator de percepção da informação técnico-científica no uso de drogas", respectivamente. Conclusões: $\mathrm{O}$ alfa de Cronbach encontrado para a escala como um todo foi de 0,859 , sugerindo fortemente a existência da fidedignidade do instrumento que se mostrou útil para avaliar o grau de conhecimento acerca do HIV/AIDS e o risco decorrente do desconhecimento, entre estudantes.

\section{ABSTRACT}

Objective: This paper presents results related to the psychometric properties of the Escala de atitudes frente a HIV/AIDS (Attitudes Towards HIVIAIDS Scale). Methods: The data was derived from a sample with 549 high and elementary school level students. The data was treated by Principal Components Analysis (PCA) and Factor Analysis (FA). The final analysis resulted in 5 factors, given a minimal eigenvalue 2. Factor load items lower than 0.30 were excluded. In this study, the lowest alpha observed was 0.79. Therefore, it is likely that all 47 final tool items measure the same construct: attitude towards HIV/AIDS. Results: Scores < 96 were considered "low knowledge amount on HIV/AIDS"; scores between 96 and 192 meant "moderate knowledge amount on HIVIAIDS", and scores > 192 were considered "high knowledge amount on HIV/AIDS". Factors

1 Universidade Estadual Paulista (Unesp). 


\section{Key-words}

Scales, validation study,

HIV, attitudes.
1, 2, and 3, i.e. "Technical/Scientific Information Perception General Factor"; "Technical/Scientific Information Perception Factor versus Sexuality and Prejudice"; "Technical/Scientific Information Perception Factor in Drug Abuse", respectively, were created. Conclusion: Cronbach's alpha found for the scale as a whole was 0.859 and strongly suggests reliability of the tool, as it showed useful to evaluate the knowledge amount regarding HIV/AIDS and the risk resulting from lack of knowledge among students.

\section{INTRODUÇÃO}

A infecção pelo vírus HIV, no Brasil, representa um importante problema de saúde pública. É um tema que apresenta vasta bibliografia, na qual são enfocados aspectos de caracterização dos diferentes grupos de risco, epidemiológicos (Brasil, 2002; Brasil, 2001a; Brasil, 2001b; Brasil, 1999) e de políticas de intervenção perante populações específicas (Bastos e Szwarcwald, 2000; Vieira et al., 2000; Fonseca et al., 2000; Bastos, 1995; Brasil, 2001c; Galduróz et al., 1997; Galduróz et al., 1995; Bucher, 1995; Marques et al., 1999; Pechansky et al., 2000; Brasil, 2001d; Rua e Abramovay, 2001; Brasil, 2003; Galvão, 2002; Marins et al., 2002; Fernandes, 2000) visando combater a epidemia da forma mais eficientemente possível, normatizando e preconizando modalidades de tratamento na rede pública de saúde (Brasil, 2000; Brasil, 2001e).

Fatores como sentimentos de culpa, ausência de informações e a impossibilidade de confrontar suas vivências pessoais e o que é dito sobre a AIDS e o HIV, como discutido por Parker e Aggleton (2001), parecem estar influenciando de maneira decisiva o tratamento, e, por vezes, determinando a protelação deste, bem como prejudicando a sua eficácia, como sugere Silva Filho e Sacardo (1997).

Estatísticas do Ministério da Saúde (Brasil, 2001a) indicam a existência de uma mudança no perfil da população entre 1991 e 2000, no que tange a não-observância aos cuidados que se deve ter para não haver transmissão do HIV, seja sexualmente, seja por uso de drogas.

Silva Filho (2000), preocupado com os problemas atinentes à prevenção da propagação do HIV, construiu um instrumento que ele convencionou chamar de "escala de atitudes frente ao HIV/AIDS", com 67 questões afirmativas, cada uma com cinco alternativas, quais sejam, concordo totalmente, concordo, sou indiferente, discordo, discordo totalmente.

Silva Filho (2000), neste estudo, verificou que: (1) faltam informações para os indivíduos, independentemente do seu grau de escolaridade ou formação; (2) áreas nas quais ainda são discutidas informações sobre a saúde tendem a ser menos permeadas pelo preconceito e pelo pensamento mágico; (3) as dificuldades para abordar este tema também estão presentes entre os formadores de opinião, no caso docentes, que não abordam o problema ou o abordam de maneira inadequada; (4) indivíduos com o HIV/AIDS apresentam fortes sentimentos de culpa e incertezas quanto ao ato de continuar ou não tomando os medicamentos; (5) o desconhecimento de dados da realidade promove oscilações que vão da quase total permissividade ao extremo de proibições, principalmente para os portadores da infecção pelo HIV; e (6) a prevenção, tanto primária quanto secundária ou terciária, deve versar sobre estes temas, sem a idéia preconcebida de que há clareza por parte do interlocutor a respeito do problema tratado.

Foram tomados os cuidados devidos no que tange a validade da escala, seguindo as recomendações de Shaw e Wright (1967), Dispoto (1977), Johnson e Wichern (1982), Romesburg (1984), Tabachnick e Fidell (1966), Lounsbury e Tornatzky (1977), Nunnally (1970), Anastasi (1967) e Likert (1932), principalmente a validade de face e de conteúdo por especialistas na área.

Foram estabelecidas categorias de perguntas para identificar a participação dos seguintes fatores: (1) religiosidade e práticas mágicas; (2) informações técnicas e científicas; (3) consumo de drogas ilícitas; (4) sexualidade; e (5) preconceito. Seguiu-se a pré-testagem do instrumento, tendo sido o questionário apresentado a uma amostra-piloto de 80 escolares da população-alvo postulada. Este trabalho teve por objetivo aprofundar o estudo das propriedades psicométricas subjacentes ao instrumento de Silva Filho (2000), consistindo em estudar a fidedignidade das subescalas por meio do alfa de Cronbach.

Trabalhos semelhantes realizados por Moriya, Gir, Hayashida (1994), Fernandes (1994), Figueiredo e Fioroni (1996), Pedrão et al. (2003), Gouveia et al. (2005), Marques et al. (2006) e Camargo e Botelho (2007), abordando diferentes preocupações quanto aos fatores que contribuem com comportamentos e atitudes de risco para contrair a infecção pelo HIV, ou a manutenção da qualidade de vida entre os que já o contraíram, ou, ainda, as dificuldades daqueles que são referências na formação de educadores, corroboram a importância deste tipo de estudo. Fatores semelhantes aos identificados nesta pesquisa foram considerados por Castanha, Coutinho, Saldanha, Ribeiro (2007, p. 27), que avaliaram tais fatores associados à percepção da qualidade de vida entre pessoas com infecção pelo HIV e concluíram que para os homens "a qualidade de vida está associada ao trabalho e ao apoio 
advindo da amizade. Já no grupo das mulheres, observa-se um predomínio de elementos subjetivos ao representarem a qualidade de vida associada ao amor, à felicidade e à figura de Deus, advinda da crença da religiosidade".

\section{MÉTODO}

\section{A amostra}

Retomou-se a amostra utilizada em Silva Filho (2000). A escala de atitudes, sob título "Escala de atitudes frente ao HIV/AIDS", foi aplicada em diferentes grupos: pessoas sabidamente portadoras do vírus HIV, alunos dos ensinos fundamental e médio e universitários. Todos os sujeitos portadores do HIV/AIDS possuíam, no máximo, a oitava série; por essa razão, quando a variável utilizada era o grau de escolaridade, foram agrupados com os alunos do ensino fundamental.

A amostra foi composta de 549 sujeitos, escolhidos aleatoriamente, sendo uma amostra de conveniência. Foram entrevistados alunos de uma escola estadual, da $8^{a}$ série ao $3^{\circ}$ colegial, alunos dos cursos de psicologia, biologia e história da Universidade Estadual Paulista (Unesp), Campus de Assis, e 21 portadores do HIV, doentes ou não de AIDS, estes escolhidos aleatoriamente, dentro de um universo de aproximadamente 60 indivíduos, sabidamente portadores do vírus HIV, participantes da ONG - Comitê Civil de Apoio e Prevenção à Aids.

\section{0 instrumento}

A escala de atitudes inicial foi construída com sete variáveis sociodemográficas (escola, série/ano, idade, sexo, religião, uso/não-uso de drogas ilícitas e ser/não ser portador do vírus HIV) e 67 frases, compostas por afirmativas e negativas, sendo 31 verdadeiras e 36 falsas.

As perguntas foram distribuídas segundo cinco categorias: religiosidade e práticas mágicas, informações técnicas e científicas, consumo de drogas ilícitas, sexualidade e preconceito. $\mathrm{Na}$ forma original, para cada frase foi solicitado aos sujeitos o grau de concordância ou discordância com o que estava sendo dito, de acordo com as alternativas (concordo totalmente, concordo, sou indiferente, discordo e discordo totalmente).

Neste trabalho foram atribuídos pontos para as respostas, a fim de transformar respostas qualitativas em dados quantitativos, para que estes pudessem sofrer um tratamento estatístico mais refinado. Depois de identificar nas perguntas as respostas mais adequadas ou verdadeiras, procedeu-se à atribuição de pontos que variaram de 1 a 5. Quando a afirmativa era falsa atribuiu-se a ela 1 ponto. Quanto mais se aproximava do comportamento ou de crenças desejáveis, indicando que o indivíduo possuía dados de realidade, atribuiu-se a ela 5 pontos. Para a indiferença foram atribuídos 3 pontos, fosse verdadeira ou falsa a afirmativa. Dizendo de maneira formal, cada um dos 67 itens foi avaliado em uma escala do tipo Likert de 5 pontos, desde 5 = concordo totalmente, $4=$ concordo, $3=$ sou indiferente, 2 = discordo e 1 = discordo totalmente. Os itens falsos receberam codificação inversa.

Os dados foram organizados, consoante: escola, curso, ano, sexo, religião, consumo de drogas ilícitas e ser portador do vírus HIV. Em seguida, foram analisados quanto a sua estrutura fatorial, concluindo-se pela postulação de uma escala para medir o grau de conhecimento acerca do HIV/ AIDS de estudantes, presumivelmente não-portadores da infecção e portadores da infecção pelo HIV.

Em um estudo semelhante, Moriya et al. (1994) construíram uma escala, inicialmente com 129 itens, que, depois de análises estatísticas, ficou reduzida a 25 itens, para verificar atitudes favoráveis e desfavoráveis diante da AIDS, com questões abrangendo a origem da doença no que diz respeito a causas, o conhecimento sobre a AIDS e a percepção do portador da infecção acerca dos relacionamentos. A amostra consistiu de jovens que freqüentavam o Tiro de Guerra e alunos do ensino médio e não avaliou portadores da infecção pelo HIV.

\section{O procedimento}

Para a aplicação do instrumento, os alunos foram convidados a participar da pesquisa. A aplicação foi realizada de maneira coletiva em diferentes dias de aulas nas respectivas salas de aulas. Os indivíduos que eram portadores da infecção pelo HIV também foram convidados a participar da pesquisa e responderam ao instrumento, na ONG que freqüentavam na época, após os esclarecimentos necessários.

\section{RESULTADOS}

O perfil sociodemográfico mostrou que $63,4 \%$ dos respondentes eram do sexo feminino e $36,6 \%$ do sexo masculino. A idade variou de 13 a 51 anos, com média de 19 anos e 2 meses e desvio-padrão de 5 anos e 2 meses.

Observou-se que $56,2 \%$ dos respondentes se manifestaram católicos, 10,5\% evangélicos, 25,2\% sem nenhuma religião e $8,1 \%$ de outras religiões. A maioria absoluta dos entrevistados $(84,6 \%)$ não se mostrou usuário de drogas ilícitas contra $14,9 \%$ de usuários. Todos os indivíduos portadores do HIV/AIDS possuíam, no máximo, a 8a série, por esta razão foram agrupados com os alunos do ensino fundamental, quando a variável utilizada era o grau de escolaridade. Segundo o grau de instrução observou-se que cerca de $48 \%$ dos respondentes eram universitários, 37\% possuíam o ensino médio e $15 \%$ o ensino fundamental.

Foram feitas duas análises estatísticas das propriedades psicométricas da escala, utilizando a análise fatorial por componentes principais no pacote estatístico SPSS-13 Statis- 
cal Package for the Social Sciences. Constatou-se que todas as suposições básicas necessárias à análise multivariada foram satisfeitas pelos dados. Optou-se pela chamada rotação Varimax, eigenvalue $=1$. A primeira análise resultou a extração de 20 fatores, agrupando-se, em geral, em um fator, as questões com afirmações positivas, negativas e intimamente relacionadas, por exemplo, o fator 11 juntou os itens: "3. AIDS é um castigo de Deus", "4. AIDS não é um castigo de Deus", "1. Deus pode curar a AIDS e ninguém precisa tomar remédios" "6. já que a AIDS não tem cura, não é preciso tomar remédios". Em um momento posterior, para verificar uma maior associação entre os itens, realizamos uma segunda análise fatorial, nas mesmas condições que a anterior, com a única diferença de tomarmos o eigenvalue mínimo como 2. Resultou agora cinco fatores, explicando 31,013\% da variância total. A primeira tabela emergente do output "SPSS" mostra os eigenvalue depois da rotação, para cada um dos fatores e as respectivas porcentagens de explicação da variância.

Tabela 1. Eigenvalues depois da rotação Varimax para os cinco fatores e porcentagens de explicação da variância e respectivas porcentagens cumulativas

\begin{tabular}{lccccc}
\hline & & Depois da rotação & \\
\cline { 2 - 3 } \cline { 5 - 5 } \cline { 5 - 5 } Componentes (fatores) & Total & & \% da variância & & \% cumulativa \\
\hline 1 & 6,590 & & 9,835 & 9,835 \\
2 & 4,238 & & 6,326 & & 16,161 \\
3 & 3,829 & & 5,715 & 21,876 \\
4 & 3,313 & & 4,944 & \\
5 & 2,809 & & 4,193 & \\
\hline
\end{tabular}

O fator 1 (componente 1), por exemplo, mostrou eigenvalue 6,590 explicando 9,835\% da variância total, e assim por diante. Constata-se que os fatores, sozinhos, estão explicando pequenas proporções da variância total. Este fato, infelizmente, ocorre com certa freqüência dentro do campo das ciências do comportamento, talvez pela grande complexidade das variáveis próprias dele.

A seguir buscamos nas subescalas (fatores ou componentes) quais seriam aqueles itens discriminativos, com a eliminação dos itens julgados "fracos". Adotamos dois critérios: (1) eliminar itens que mostrassem carga fatorial menor que 0,30, em valor absoluto, segundo critério de Hair et al. (1998), e (2) encontrar a consistência interna de cada um dos fatores (ou subescalas) pelo coeficiente alfa de Cronbach.

Neste estudo, o menor alfa observado foi de 0,79. Pavot e Diener (1993) descrevem como ótimos os coeficientes no intervalo 0,79 e 0,89. Portanto, é muito provável que todos os itens do instrumento final elaborado meçam o mesmo construto: atitude diante do HIV/AIDS.

Observando a Rotated Component Matrix, obtida pela SPSS (2001), descartamos imediatamente três itens, por mostrarem cargas muito baixas nos cinco fatores: q10 (a saliva mata o vírus causador da AIDS), q36 (doar sangue pode transmitir HIV/AIDS) e q52 (as pessoas não gostam de quem tem AIDS). Eliminamos os fatores 4 e 5 por terem apresentado baixos índices de consistência interna, respectivamente, alfa $=0,43,7$ itens e alfa $=0,578,9$ itens.

Assim, a escala devidamente revalidada, ficou com 47 itens. A análise fatorial final mostrou: o fator 1 agrupando 24 itens, explicando 9,835\% da variância total, com um índice de consistência alfa $=0,843$. Eis o quadro do fator 1 :

Quadro 1. Fator 1: fator geral de percepção da informação técnico-científica, variáveis com pesos e descrições explicando 9,835\% da variância total (alfa $=0,843$ )

\begin{tabular}{|c|c|c|}
\hline Variável & Peso & Descrição da variável \\
\hline q21 & 0,569 & Não se contrai AIDS por um abraço. \\
\hline q22 & 0,550 & Quem tem AIDS não precisa usar camisinha. \\
\hline q41 & 0,540 & Não se contrai AIDS por um aperto de mão. \\
\hline q1 & 0,538 & Deus pode curar a AIDS e ninguém precisa tomar remédios. \\
\hline q30 & 0,537 & $\begin{array}{l}\text { AIDS é uma doença que atinge apenas pessoas de } \\
\text { determinados grupos de risco. }\end{array}$ \\
\hline q8 & 0,525 & $\begin{array}{l}\text { Coquetel não cura e por isso não é necessário tomá-lo } \\
\text { para não ter doenças oportunistas. }\end{array}$ \\
\hline q33 & 0,522 & A transfusão de sangue não transmite 0 HIV/AIDS. \\
\hline$q 63$ & 0,482 & AIDS é uma doença que não atinge apenas homossexuais. \\
\hline q6 & 0,472 & Já que AIDS não tem cura, não é preciso tomar remédios. \\
\hline q18 & 0,457 & $\begin{array}{l}\text { A AIDS não pode ser contraída em dentistas, ginecologistas, } \\
\text { cabeleireiros e manicures. }\end{array}$ \\
\hline q23 & 0,448 & A saliva não mata o vírus causador da AIDS. \\
\hline q26 & 0,436 & AIDS é uma doença que atinge apenas homossexuais. \\
\hline q20 & 0,434 & Pode-se contrair AIDS por um aperto de mão. \\
\hline q58 & 0,430 & Pode-se contrair AIDS por um abraço. \\
\hline q16 & 0,397 & $\begin{array}{l}\text { Coquetel não cura, mas é necessário tomá-lo para } \\
\text { não ter doenças oportunistas. }\end{array}$ \\
\hline$q 62$ & 0,388 & $\begin{array}{l}\text { A AIDS pode ser contraída nos consultórios de dentistas e } \\
\text { ginecologistas, e também em cabeleireiros e manicures. }\end{array}$ \\
\hline q48 & 0,386 & Suor não pode transmitir HIV/AIDS. \\
\hline q56 & 0,386 & AIDS é uma doença que pode atingir qualquer pessoa. \\
\hline q4 & 0,386 & AIDS não é um castigo de Deus. \\
\hline q31 & 0,374 & Não se contrai AIDS pela picada de insetos. \\
\hline q61 & 0,364 & Mesmo a AIDS não tendo cura, é necessário tomar os remédios. \\
\hline q3 & 0,356 & AIDS é um castigo de Deus. \\
\hline q25 & 0,339 & Quem tem AIDS precisa usar camisinha. \\
\hline$q 65$ & 0,320 & A transfusão de sangue pode transmitir o HIV/AIDS. \\
\hline
\end{tabular}

Este fator parece agrupar predominantemente os itens relativos às informações gerais que poderiam contribuir com atitudes influenciadas por religiosidade e práticas mágicas, informações técnicas e/ou científicas, pelo uso de drogas ou pela impressão sobre o uso destas, pela forma de encarar a sexualidade quando houvesse a manifestação do HIV e por preconceitos determinados por valores pensados de uma 
forma mais ampla. Por essas razões chamaremos o fator 1 de "fator geral de percepção da informação técnico-científica".

O fator 2 agrupa 12 itens e explica 4,24\% da variância total. Esta subescala mostrou um índice de consistência interna alfa $=0,786$. A seguir o quadro do fator 2 :

Quadro 2. Fator 2: fator de percepção da informação técnicocientífico versus sexualidade e preconceito, variáveis com pesos e descrições explicando 4,24\% da variância total $($ alfa = 0,786)

\begin{tabular}{|c|c|c|}
\hline Variável & Peso & Descrição da variável \\
\hline$q 60$ & 0,728 & Pode-se contrair AIDS pela saliva. \\
\hline q19 & 0,689 & Pode-se contrair AIDS pelo beijo. \\
\hline q34 & 0,662 & Não se contrai AIDS pelo beijo. \\
\hline q67 & 0,583 & Não se pode contrair AIDS pela saliva. \\
\hline q47 & 0,548 & $\begin{array}{l}\text { Se contrai HIV/AIDS por meio de copos, } \\
\text { talheres, pratos e roupas de cama. }\end{array}$ \\
\hline q11 & 0,53 & Pode-se contrair AIDS pelo uso de sanitários públicos. \\
\hline q40 & 0,475 & Pode-se contrair AIDS por picada de insetos. \\
\hline q2 & 0,441 & Não se contrai AIDS pelo uso de sanitários públicos. \\
\hline q13 & 0,418 & $\begin{array}{l}\text { Não se contrai HIV/AIDS por meio de copos, talheres, } \\
\text { pratos e roupas de cama. }\end{array}$ \\
\hline q54 & 0,408 & $\begin{array}{l}\text { Não devemos nos aproximar de pessoas portadoras de AIDS, } \\
\text { pois existe risco de contaminaçãa. }\end{array}$ \\
\hline q7 & 0,384 & Suor pode transmitir HIV/AIDS. \\
\hline q51 & 0,369 & $\begin{array}{l}\text { Podemos nos aproximar de pessoas portadoras de AIDS, } \\
\text { pois não existe risco de contaminação. }\end{array}$ \\
\hline
\end{tabular}

Este fator 2 leva em conta predominantemente a existência de preconceitos e a percepção e a forma de viver a sexualidade. Recebe, portanto o nome de: "fator de percepção da informação técnico-científica versus sexualidade e preconceito".

O fator 3 agrupa 11 itens e explica 3,829\% da variância total. Esta subescala mostrou um índice de consistência interna alfa $=0,789$. A seguir o quadro do fator 3 :

Quadro 3. Fator 3: fator de percepção da informação técnicocientífica no uso de drogas, variáveis com pesos e descrições explicando 3,829\% da variância total (alfa $=0,789)$

\begin{tabular}{lrl}
\hline Variável & Peso & \multicolumn{1}{c}{ Descrição da variável } \\
\hline q27 & 0,657 & Cocaína faz mal para quem tem AIDS. \\
q43 & 0,644 & Maconha não faz mal para quem tem AIDS. \\
q38 & 0,611 & Álcool faz mal para quem tem AIDS. \\
q12 & 0,61 & Maconha faz mal para quem tem AIDS. \\
q53 & 0,586 & Álcool não faz mal para quem tem AIDS. \\
q64 & 0,545 & Cocaína não faz mal para quem tem AIDS. \\
q32 & 0,532 & Crack não faz mal para quem tem AIDS. \\
q66 & 0,528 & Crack faz mal para quem tem AIDS. \\
q9 & 0,505 & As drogas não fazem mal para ninguém. \\
q50 & 0,47 & Crack faz mal para qualquer pessoa. \\
q5 & 0,464 & As drogas fazem mal. \\
\hline
\end{tabular}

O fator 3, por envolver a percepção de drogas, podendo inclusive ocorrer por uso, denominou-se: "fator de percepção da informação técnico-científica no uso de drogas".

O alfa de Cronbach encontrado para a escala como um todo foi de 0,859 . Os dados sugerem fortemente a existência da fidedignidade do instrumento.

As médias e desvios-padrão para as escalas e subescalas são apresentadas na tabela 2, a seguir.

Tabela 2. Médias e desvios-padrão para as subescalas e escala total

\begin{tabular}{lcccc}
\hline Escalas & Mínimo & Máximo & Média & Desvio-padrão \\
\hline Fator 1 & 25 & 125 & 106,80 & 11,770 \\
Fator 2 & 12 & 60 & 47,68 & 7,436 \\
Fator 3 & 11 & 55 & 43,58 & 6,807 \\
Escala Total & 48 & 240 & 198,06 & 18,648 \\
\hline
\end{tabular}

Fator 1: fator geral de percepşão da informação técnico-científica; fator 2: fator de percepçăo da informação técnico-científico versus sexualidade e preconceito; fator 3: fator de percepção da informaçăo técnico-científica no uso de drogas.

As diferenças observadas entre o fator 1 (fator geral de percepção da informação técnico-científica) e o fator 2 (fator de percepção da informação técnico-científica versus sexualidade e preconceito) parecem existir em virtude de o fator 1 ser menos específico que o fator 2, que concentra itens relacionados predominantemente ao preconceito, enquanto o fator 1 implica informações técnico-científicas, podendo em algumas situações o preconceito ser confundido com ignorância.

Observe-se que o padrão dos dados, tanto nas subescalas como na escala, é absolutamente o mesmo: o agrupamento da maioria em torno da média está mais próximo do valor máximo das subescalas ou da escala, conforme o caso.

Entendemos, finalmente, que seja útil trabalhar com a escala como se ela fosse qualitativa, encontrando-se os pontos de corte 96 e 192 e decidindo-se que: (1) aqueles sujeitos, cujo perfil sociodemográfico foi estabelecido no início, que obtiverem um escore inferior a 96 estarão em uma categoria rotulada como "fraco grau de conhecimento sobre HIV/AIDS"; (2) os que obtiverem escores entre 96 e 192 estarão em uma categoria rotulada como "moderado grau de conhecimento" e (3) os que obtiverem escores acima de 192 estarão na categoria "alto grau de conhecimento sobre HIV/AIDS". A grande maioria dos respondentes do estudo recai na terceira categoria.

\section{DISCUSSÃO}

Este trabalho teve como objetivo, entre outros, analisar os aspectos psicométricos que estão por trás de um instrumento formulado por Silva Filho (2000) com o fim precípuo de estudar as atitudes de estudantes e portadores da infecção diante do HIV/AIDS. O instrumento apresentou proprie- 
dades psicométricas bem definidas, podendo ser utilizado por profissionais que estejam interessados em avaliações específicas dentro de um contexto populacional com o perfil do estudado.

$\mathrm{Na}$ análise fatorial utilizando o método das componentes principais, adotamos o critério comumente aceito de só levar em conta as variáveis com cargas fatoriais maiores que 0,30. Por meio dessa análise estudamos a validade de construto para o instrumento. A consistência interna encontrada, tanto para a escala como um todo quanto para cada subescala em particular, forneceu índices que podem ser considerados coerentes com os atuais parâmetros usualmente aceitos. Após as análises estatísticas a escala ficou com 47 itens dos 67 iniciais.

Um problema, porém, parece ter ocorrido com as proporções pequenas da variância total que foram explicadas pelos três fatores, respectivamente, 9,835\%, 4,24\% e 3,829\%. Embora muitos especialistas nas áreas de ciências humanas, principalmente em psicologia, afirmem que tal fato geralmente ocorre em razão da complexidade das variáveis próprias do campo da psicologia, entendemos que novas pesquisas carecem ser realizadas, para que se tenham resultados mais conclusivos.

\section{CONCLUSÕES}

O fator 1 (fator geral de percepção da informação técnicocientífica) permitiu agrupar itens relativos às informações gerais que poderiam contribuir com atitudes influenciadas por religiosidade e práticas mágicas, informações técnicas e/ou científicas, pelo uso de drogas ou pela impressão sobre o uso destas, pela forma de encarar a sexualidade quando o HIV se manifesta e por preconceitos determinados por valores pensados de uma forma mais ampla.

O fator 2 (fator de percepção da informação técnicocientífica versus sexualidade e preconceito) mostrou a existência de preconceitos e a percepção e a forma de viver a sexualidade.

O fator 3 (fator de percepção da informação técnicocientífica no uso de drogas) permitiu avaliar atitudes favoráveis e desfavoráveis diante do consumo de drogas, com implicações negativas na ocorrência da infecção pelo HIV. O alfa de Cronbach encontrado para a escala como um todo foi de 0,859 , sugerindo fortemente a existência da fidedignidade do instrumento.

Os sujeitos com perfis sociodemográficos, semelhantes ao estudado nesta pesquisa, que obtiverem um escore inferior a 96 estarão em uma categoria rotulada como "fraco grau de conhecimento sobre HIV/AIDS" e, portanto, vulneráveis; os que obtiverem escores entre 96 e 192 estarão em uma categoria rotulada como "moderado grau de conhecimento" e, portanto, com possibilidade de comportamentos de risco; e, os que obtiverem escores acima de 192 estarão na categoria "alto grau de conhecimento sobre HIV/AIDS" e, portanto, menos vulneráveis a comportamentos de risco para contrair a infecção e/ou emitir comportamentos, fundados em preconceitos, diante de indivíduos portadores da infecção.

\section{REFERÊNCIAS}

Anastasi A. Testes Psicológicos: teoria e aplicação. São Paulo, Herder, 1967.

Bastos IF, Szwarcwald CL. Aids e Pauperização: Principais Conceitos e Evidências Empíricas, Cadernos de Saúde Pública, 16:65-76, 2000.

Bastos IF. Limitações estruturais a implantação de estratégias preventivas relativas à disseminação do HIV entre usuários de drogas injetáveis no Brasil. In: Czeresnia D, Santos EM, Barbosa RHS. Aids: Pesquisa Social e Educação. Rio de Janeiro: Hucitec, p.166-192, 1995.

Bucher R. 0 usuário de drogas injetáveis na política preventiva ao HIV/AIDS. In: Czeresnia D, Santos E M, Barbosa RHS. Aids: Pesquisa Social e Educação. Rio de Janeiro: Hucitec, p. 147-166, 1995.

Brasil, Ministério da Saúde. Ms divulga novos dados da AIDS e anuncia índices de resistência do HIV aos anti-retrovirais, [serial online], 2003; [cited 2003 maio 16] available from: http:// www.AIDS.gov.br/ final/ imprensa1/ imprensa.HTM

Brasil, Ministério da Saúde. Boletim Epidemiológico Aids.Brasília:Ministério da Saúde, v 16, p. 1-45. [semana ep. 14a a 52a], 2002.

Brasil, Ministério da Saúde. Boletim Epidemiológico Aids.Brasília:Ministério da Saúde, v.15, p.159. [semana ep. 27a a 40a], 2001a.

Brasil, Ministério da Saúde. Coordenação Nacional de DST e AIDS. Manual de Redução de Danos: Saúde e Cidadania, Série Manuais, v.49, p. 1-114, 2001b.

Brasil, Ministério da Saúde. Coordenação Nacional de DST e AIDS. Projeto Ajude-Brasil: Avaliação epidemiológica dos usuários de drogas injetáveis dos Projetos de Redução de Danos (PRD) apoiados pela CN-DST/AIDS. Brasília, p. 1-341, 2001C

Brasil, Ministério da Saúde. Boletim Epidemiológico Aids.Brasília:Ministério da Saúde, v. 12, p.157. [semana ep. 22ª 34ª], 1999.

Camargo, Brigido V, Botelho, Lúcio J. Aids, Sexuality and attitude of adolescents about protection against HIV. Rev. Saúde Pública, São Paulo, v. 41, n.1,2007. [disponível em: http:// www.scielo.br/scielo.php? scrip t=sci_arttext\&pid= s003 4- 89102007000100009\& Ing=en\&nrm=iso>]. Acesso em: 21 may 2007. Pré-publicação. doi: 10.1590/s003489102007000100009

Castanha RA, Coutinho MPL, Saldanha AAW, Ribeiro CG. Avaliação da qualidade de vida em soropositivos para o HIV. Estudos de Psicologia, 24(1); 23-32, 2007.

Dispoto RG. Interrelationships among measures of environmental activity, emotionality, and knowledge. Educational and psychological measurement, 37; 451-459, 1977.

FERNANDES, J. C. L. Práticas educativas para a prevenção do HIV/AIDS: Aspectos conceituais, Cadernos Saúde Pública, Rio de Janeiro, 10(2): 171-180, 1994

Fernandes MAS, Antonio DG, Bahamondes LG, Cupertino CV. Conhecimento, atitudes e práticas de mulheres brasileiras atendidas pela rede básica de saúde com relação às doenças de transmissão sexual, Cadernos de Saúde Pública, 16;103-112, 2000.

Figueiredo MAC, Fioroni LN. Atitudes frente à AIDS e lócus de controle: um estudo com estudantes e profissionais de enfermagem. Medicina, Ribeirão Preto, v. 29, n. 3 e 4, p.301-308, 1996.

Fonseca GM, Bastos Fl, Derriço M, Andrade CLT, Travassos C, Szwarcwald CL. Aids e grau de escolaridade no Brasil: evolução temporal de 1986 a 1996. Cadernos de Saúde Pública, $16 ; 77-87,2000$

Galduróz JCF, Noto RA, Carlini EA. IV Levantamento sobre o uso de drogas entre estudantes de $1^{\circ}$ e $2^{\circ}$ Graus em 10 capitais brasileiras - 1997; São Paulo:Universidade Federal de São Paulo; Centro Brasileiro de Informações Sobre Drogas Psicotrópicas - Cebrid; 1997.

Galduróz FCJ, Noto AR, Carlini AE. A adolescência, o ensino e o abuso de drogas: reflexões. Temas, 49; 48-57, 1995 
Galvão MTG, Aplicação do Instrumento HAT-Q0L para análise da qualidade de vida de mulheres com infecção pelo HIV, ou com AIDS e sua correlação com as variáveis sócio-demográficas, epidemiológicas e clínicas. [Tese] Botucatu: Unesp; 2002.

Gouveia VV, Pimentel CE, Queiroga F, Meira M, Jesus RG. Escala de atitudes frente ao uso de maconha: comprovação da sua validade de construto, Jornal Brasileiro de Psiquiatria, 54 (1):5-12, 2005.

Hair, J. F.; Anderson, R.E.; Tatham, R.L; Black, W.C. Multivariate data analysiz. Fifth Edition. New Jersey. Prentice Hall, 1998.

Johnson, R. A. \& Wichern, D. W. Applieed multivariate statistical analysis. Englewood Cliffs, Prentice-Hall, 1982.

Likert, R. A technique for the measurement of attitudes. Arch. Psychol, 140:1-55, 1932.

Lounsbury JW, Tornatzky LG. A scale for assessing attitudes toward environmental quality. The Journal of Social Psychology, 101:299-305, 1977.

Marques LF, Doneda D, Serafin D. 0 uso indevido de drogas e a AIDS. in: Brasil, Ministério da Saúde, Brasília, Secretária de Políticas de Saúde. Cadernos Juventude Saúde e Desenvolvimento, 1:173-183, 1999.

Marques HHS E COL., A revelação do diagnóstico na perspectiva dos adolescentes vivendo com HIV/AIDS e seus pais e cuidadores, Cadernos de Saúde Pública, Rio de Janeiro, 22(3):619629,2006

Marins PRJ et al. Relatório de pesquisa: sobrevivência atual dos pacientes com AIDS no Brasil. Evidência dos resultados de um esforço nacional. Boletim Epidemiológico AIDS. Brasília: Ministério da Saúde. [Serial Online] [cited 2002 abr 21]. avaiable from: http:// www.AIDS. gov.br/final/biblioteca/bol_marco_2002/artig01.htm

Moriya MT, Gir E, Hayashida M. Escala de atitude frente à AIDS: uma análise psicométrica, Revista Latino-Americana de Enfermagem, 2:37-53, 1994.

Nunnally JC. Introduction to psycological mensurement. New York, McGraw-Hill, 1970.

Parker R, Aggleton P. Coleção ABIA, Cidadania e Direitos, Estigma, Discriminação e Aids, Rio de Janeiro, n.1, 2001
Pavot W, Diner E. Review of the satisfaction with life scale. Psychological Assesment, 5(1):6472, 1993.

Pechansky F, Inciardi AJ, Surratt H, Lima AFBS et al. Estudo sobre as características de usuários de drogas injetáveis que buscam atendimento em Porto Alegre, RS. Rev Bras Psiquiatr, 22:164-171, 2000.

Pedrão LJ, Avanci RC, Malaguti SE, Aguilera AMS. Atitudes frente à doença mental: estudo comparativo entre ingressantes e formandos em enfermagem, Medicina, Ribeirão Preto, 36:37-44, 2003

Romesburg HC. Cluster analysis for researchers, Lifetime Learning Publications, Belmont, California, 1984.

Rouselle DM. Adolescents' perceptions of factors affecting their decisions to seek health care. Journal of American M Assoc, 273(24):1913-1918, 1995.

Rua GM, Abramovay M. Avaliação das ações de prevenção às DST/AIDS e uso indevido de drogas nas escolas de ensino fundamental e médio em capitais brasileiras. Brasília, Unesco, 2001.

Shaw EM, Wright JM. Scales for the mensurement of attitudes. New York, McGraw-Hill, 1967.

Silva Filho N. Estudo de fatores determinantes de atitudes de estudantes do ensino fundamental, médio e superior: identificação de comportamentos estigmatizantes entre não portadores do HIV/AIDS frente aos portadores e entre os portadores, Relatório Científico, Unesp, Campus de Assis, Departamento de Psicologia Clínica, 2000.

Silva Filho N, Sacardo DP. Estudo longitudinal da variação da eficácia adaptativa e do equilíbrio adaptativo de pacientes portadores de HIV/AIDS e evoluções em psicoterapia. in: II Jornada de Pesquisa, Assis. A pesquisa no Campus: Diagnóstico e Reflexão. Assis: Gráfica da Faculdade de Ciências e Letras - UNESP, Campus de Assis, 1:32-33, 1997.

SPSS - Statistical package for the social sciences, Chicago: SPSS, 2001.

Tabachnick GB, Fidell LS. Using multivariate statistics. New York: Harrer Collins, 1966.

Vieira ME, Villela WV, Réa MF, Fernandes MEL, Franco E, Ribeiro G, Alguns aspectos do comportamento sexual e prática de sexo seguro em homens do Município de São Paulo, Cadernos de Saúde Pública, 16: 997-1009, 2000. 